\title{
AUTONOMIA EM IDOSOS COM DOENÇA DE ALZHEIMER: CONTRIBUIÇÕES DO PROJETO ESTRATÉGIAS DE DIAGNÓSTICO EREABILITAÇÃO SOCIAL DE IDOSOS DEPENDENTES E APOIO PSICOSSOCIAL DE CUIDADOR DOMICILIAR
}

\author{
Marilia de Rosso Krug ${ }^{1}$ \\ Karine Bueno do Nascimento ${ }^{2}$ \\ Solange Beatriz Billig Garces ${ }^{3}$ \\ Carolina Böettge Rosa ${ }^{4}$ \\ Ângela Vieira Brunellis \\ Dinara Hansen ${ }^{6}$
}

\footnotetext{
1 Licenciada em Educação Física. Mestre em Ciências do Movimento Humano. Professora Adjunta da Universidade de Cruz Alta (UNICRUZ), vinculada ao curso de Educação Física. E-mail: mkrug@unicruz.edu.br.

2 Graduada em Educação Física. Mestre em Educação em Ciências.

E-mail: karinebueno20@hotmail.com.

3 Licenciada em Educação Física. Doutora em Ciências Sociais. Professora Adjunta ll da Universidade de Cruz Alta (UNICRUZ). Líder do Grupo Interdisciplinar de Estudos do Envelhecimento Humano (GIEEH). E-mail: sgarces@unicruz.edu.br.

4 Graduada em Nutrição. Mestre em Patologia. Doutoranda em Gerontologia Biomédica. Pesquisadora do Grupo Interdisciplinar de Estudos do Envelhecimento Humano da UNICRUZ. E-mail: carolboettge@gmail.com.

5 Graduada em Nutrição. Mestre em Extensão Rural. Professora adjunta da Universidade de Cruz Alta (UNICRUZ), vinculada ao Centro de Ciências da Saúde e Agrárias. E-mail: abrunelli@unicruz.edu.br.

6 Graduada em Fisioterapia. Mestre em Gerontologia Biomédica. Professora Assistente da Universidade de Cruz Alta (UNICRUZ), vinculada ao Centro de Ciências da Saúde e Agrárias. E-mail: dhansen@unicruz.edu.br.
} 
resumo

Este estudo buscou analisar as contribuições de atividades terapêuticas psicossociais e funcionais na autonomia e na aptidão física de seis idosos com doença de Alzheimer. A autonomia foi avaliada com a Escala ADL de Katz e a Aptidão física através da bateria de testes de Rikli e Jones. Os encontros com o grupo de idosos e cuidadores ocorreram quinzenalmente, perfazendo um total de 12 sessões. As atividades terapêuticas psicossociais e funcionais constaram de alongamentos, caminhada e dança, tendo cada sessão duração de aproximadamente 40 minutos. Na sequência, os idosos realizavam atividades diversificadas de memorização através de jogos didáticos, quebra-cabeças, jogos de habilidades motoras finas e destrezas manuais, além de atividades sensoriais envolvendo gosto, tato e olfato. Os dados foram interpretados e descritos por meio da inferência percentual e da estatística descritiva. Após analisar os dados, observaram-se melhoras, de pré para pós-teste, nas variáveis: capacidade de transferir-se de um local para outro, em 6,7\%, na continência, em 16,7\%, e na capacidade para alimentar-se, em $66,7 \%$, sendo esta última estatisticamente significativa ( $p=0,025)$. Os valores da aptidão física mostraram que os idosos estavam numa classificação muito abaixo do normal. Ao comparar esses valores em termos de média, de pré para o pós-teste, foi possível observar melhora estatisticamente significativa $(p=0,012)$ somente na variável de flexibilidade de membros superiores. Sendo assim, foi possível concluir que um programa de atividades físicas para idosos com Alzheimer é muito importante, no entanto deve haver maior regularidade de sessões para que os resultados possam ser mais relevantes.

palavras-chave

Doença de Alzheimer. Atividade Motora. Aptidão Física.

De acordo com o relatório sobre a situação da população mundial (ONU, 2011) uma em cada nove pessoas no mundo tem 60 anos ou mais (o equivalente a $11,5 \%$ da população mundial) e o envelhecimento, embora seja um fenômeno comum a nações ricas e pobres, está aumentando mais rapidamente nos países em desenvolvimento, onde vivem 2 de cada 3 pessoas idosas. $\mathrm{Na}$ 
última década, o número de idosos no mundo aumentou em 178 milhões de pessoas. A cada segundo, 2 pessoas celebram o 60. aniversário. No Brasil, para o ano de 2060, estima-se que o número de idosos deva chegar a 75,1 milhões, representando 32,9\% da população brasileira, ou seja, 1 em cada 3 brasileiros terá 60 anos ou mais (ALVES, 2014).

Com o crescimento da população idosa, aumenta também a incidência de doenças crônicas não transmissíveis (DCNT). No cenário brasileiro, 85\% dos idosos possuem pelo menos uma doença crônica e 10\% destes apresentam comorbidades (SANTOS; PAVARINI, 2010).

Dentre as DCNT que acometem os indivíduos ao longo do processo de envelhecimento, as demências se destacam por terem características que não afetam apenas o indivíduo doente, mas se estendem a toda estrutura familiar e à sociedade, causando nelas um grande impacto psicossocial e econômico (ARRUDA; ALVAREZ; GONÇALVES, 2008).

Dentre as demências, a doença de Alzheimer (DA) é a que mais acomete os idosos, tornando-os incapacitados para a realização das atividades básicas da vida diária (OLIVEIRA et al., 2005), constituindo-se, assim, como uma problemática de saúde pública em todo o mundo. O percentual de idosos acometidos pela DA aumenta conforme a idade. Afeta cerca de $9 \%$ da população com mais de 65 anos, 34\% daqueles com mais 85 anos e até 43\% daqueles com 95 anos (VIEIRA; CAIXETA, 2012).

De acordo com o relatório de 2012 da Organização Mundial da Saúde (OMS), atualmente mais de 35 milhões de pessoas em todo o mundo têm demência, número que deverá duplicar em 2030 (66 milhões) e triplicar até 2050 (115 milhões). No Brasil, não existem dados precisos sobre quantas pessoas têm a DA, mas, segundo a OMS, estima-se que sejam aproximadamente 1,2 milhões de casos desta demência (WHO, 2012), já para o estado do Rio Grande do Sul, a estimativa de pessoas com a demência passa dos 58 mil habitantes (COUTINHO FILHO, 2008).

A DA tem particular importância devido às limitações que impõe ao doente, agravando todas aquelas perdas já esperadas com o envelhecimento. Essa doença provoca perda progressiva das habilidades de raciocinar e memorizar e afeta as áreas cerebrais relacionadas à linguagem e a capacidade para cuidar de si mesmo, produzindo grande dependência de parentes e de profissionais de saúde, principalmente, na fase final da doença (ABREU; FORLENZA; BARROS, 2005). A DA não afeta somente a pessoa acometida, mas sim a todos os que estão ao seu redor, pois com a progressão da doença, o idoso fica cada vez mais dependente do seu cuidador, que neste caso tem sua vida modificada para poder atender às necessidades básicas do idoso com Alzheimer (SCHLINDWEIN-ZANINI, 2010). 
Segundo Bandeira, Gonçalves e Pawlowski (2007), dentre essas necessidades, além das já esperadas com o envelhecimento, podemos citar a apraxia (incapacidade de executar movimentos voluntários coordenados), a afasia (habilidade deficiente em compreender a linguagem nas suas formas escrita ou falada) e a agnosia (perda da capacidade de reconhecer objetos). Segundo os autores supracitados, ocorrem também uma mudança de comportamento e de personalidade e dificuldades de ordem afetiva dos idosos com DA.

Diante do quadro apresentado, Matsudo (2009) salienta o papel fundamental da atividade física no processo de envelhecimento, independentemente de ter ou não a DA, pois aquela tem significância na prevenção e no controle das doenças crônicas não transmissíveis e apresenta vários efeitos benéficos, tais como: fortalecimento da massa muscular, aumento do volume sanguíneo circulante, diminuição da frequência cardíaca, melhora da autoestima, prevenção ou retardo do declínio das funções cognitivas, diminuição do estresse, ansiedade e depressão.

Segundo Zidan et al. (2012), o exercício físico está associado à menor prevalência e incidência de demência, bem como ao declínio cognitivo. Percebe-se que a realização de exercícios generalizados (resistência, mobilidade e coordenação) pode diminuir a deterioração no desempenho das AVD significativamente, aumentando a capacidade funcional global e a habilidade para desempenhar as AVD (ZIDAN et al., 2012).

Considerando a importância de intervenções que minimizem as consequências da DA e que contribuam para a autonomia e a qualidade de vida dos idosos acometidos por esta doença, foi proposto pelo Grupo Interdisciplinar de Estudos do Envelhecimento Humano da Universidade de Cruz Alta (GIEEH/ UNICRUZ), no Rio Grande do Sul, um projeto de extensão que oferecesse sessões terapêuticas psicossociais e funcionais para idosos com Alzheimer e seus respectivos cuidadores (GARCES et al., 2010). Dessa forma, este estudo teve como objetivo avaliar os efeitos de atividades terapêuticas psicossociais e funcionais na autonomia e na aptidão física de idosos com a DA.

\section{Material e métodos}

Participaram, deste estudo de caso, seis (06) idosos com diagnóstico da doença de Alzheimer cadastrados no projeto de extensão Estratégias de Diagnóstico e Reabilitação Social de Idosos Dependentes e Apoio Psicossocial de Cuidador Domiciliar. Esse projeto era desenvolvido numa parceria entre a Universidade de Cruz Alta (UNICRUZ) e a Prefeitura Municipal de Cruz Alta, RS. 
Para avaliar a autonomia dos idosos, utilizou-se o questionário de avaliação para a Escala Activities of Daily Living (ADL, Atividades da Vida Diária) de Katz, originalmente desenvolvida para avaliar a condição funcional de idosos e doentes crônicos com dificuldades motoras (FARINATTI, 2008). O índice ADL mede, em uma escala cumulativa e dicotômica, o nível de dependência funcional em seis dimensões: banho, vestuário, higiene pessoal, transferência (cama e cadeira), continência e alimentação. Por meio da observação e de questões feitas ao paciente ou ao seu cuidador, atribuiu-se uma posição em uma escala de três pontos. Para obtenção do índice ADL, transferiu-se seu resultado para uma classificação do tipo "dependente" e "independente", o que permitiu uma classificação de 0 a 6 , na qual " 0 " representou independência e "6" dependência em todos os itens (FARINATTI, 2008).

Para a avaliação da aptidão física, utilizou-se a bateria de testes de aptidão física e saúde proposta por Rikli e Jones (2008), desenvolvidos para atender à necessidade da existência de uma bateria de testes de fácil aplicação para avaliar a aptidão física em idosos. Especificamente, os testes medem os atributos físicos, ou seja, força dos membros inferiores e superiores, resistência aeróbica, flexibilidade de membros inferiores e superiores, agilidade e equilíbrio dinâmico. Os testes aplicados, respectivamente, foram: levantar da cadeira, flexão de braços, caminhada de seis minutos, sentar e alcançar os pés, alcançar as costas e o teste de levantar e caminhar.

Tanto a escala de Katz como os testes de aptidão física foram aplicados em duas etapas caracterizadas aqui como pré e pós-teste. O pré-teste foi aplicado em março de 2010 na primeira semana de atividades do projeto, já o pós-teste foi aplicado em novembro de 2010. Os encontros com o grupo de idosos e cuidadores ocorreram quinzenalmente, perfazendo um total de 12 sessões.

As atividades terapêuticas psicossociais e funcionais foram desenvolvidas no Campus da UNICRUZ e constaram de alongamentos, caminhada e dança, caracterizadas como atividades físicas aeróbicas. Esta sessão durava aproximadamente 40 minutos, após os idosos realizarem atividades diversificadas de memorização através de jogos didáticos, quebra-cabeças, jogos de habilidades motoras finas e destrezas manuais, além de atividades sensoriais envolvendo gosto, tato e olfato. As atividades de socialização geralmente eram ministradas concomitantemente, sendo que todas estas atividades eram coordenadas por todos da equipe multidisciplinar (enfermeiras, fisioterapeutas, educadores físicos, nutricionistas e psicopedagogas) do grupo.

As atividades aeróbicas foram realizadas no ginásio de esporte e dependências externas do Campus da Universidade de Cruz Alta e foram desenvolvidas em espaço específico para a prática de exercícios, nivelado e ventilado, 
coordenada por profissionais habilitados em Educação Física e Fisioterapia. Os exercícios foram planejados e controlados quanto à intensidade com base em metodologia de exercícios físicos aeróbicos específicos para idosos segundo protocolo proposto por Pereira e Safons (2007).

Os dados foram analisados quantitativamente, atribuindo frequência simples, percentual, média e desvio padrão. Foi utilizado o teste " $\mathrm{t}$ " de Student para amostras dependentes na comparação das variáveis de aptidão física entre pré e pós-teste. O nível de significância adotado foi de $\mathrm{p} \leq 0,05$ e também qualitativamente através das observações realizadas pela equipe de trabalho anotada em diário de campo. Este estudo foi aprovado pelo Comitê de Ética em Pesquisa da Universidade de Cruz Alta, Rio Grande do Sul, com o parecer no 0017.0.417.000-11.

\section{Resultados}

Quanto ao perfil do grupo, pode-se destacar que a maioria era do sexo feminino, ou seja, o grupo era constituído por dois homens e quatro mulheres com idade média de 69,16 $\pm 3,97$ anos. Dentre os participantes, cinco (83\%) dos idosos moravam com um dos filhos, sendo que o mais jovem possuía 63 anos e o mais velho, 90 anos. Com referência à ocupação, entre as mulheres, a maioria trabalhava como dona de casa e uma delas era professora; entre os homens, um era tropeiro (condutores de tropas ou comitivas de muares e cavalos) e o outro realizava serviços gerais. Todos os idosos fizeram parte do grupo experimental.

A autonomia dos idosos foi avaliada considerando a condição funcional deles em realizar seis distintas atividades básicas da vida diária (ABVD) que foram classificados em independentes e dependentes. Nesta categoria, se inclui os dependentes parciais e totais, de acordo com o proposto pela Escala Activities of Daily Living (ADL, Atividades da Vida Diária) de Katz (FARINATTI, 2008) que pode ser visto na Tabela 1.

Tabela 1 - Condição funcional de pré e pós-teste.

\begin{tabular}{l|c|c|c|c}
\hline \multirow{2}{*}{ ABVD } & \multicolumn{4}{|c}{ Classificação (\%) } \\
\cline { 2 - 5 } & \multicolumn{2}{|c}{ Pré-teste (\%) } & \multicolumn{2}{c}{ Pós teste (\%) } \\
\cline { 2 - 5 } & Ind. & Dep. & Ind. & Dep. \\
\hline Banho & 16,7 & 83.3 & 0 & 100 \\
\hline Vestuário & 0 & 100 & 0 & 100 \\
\hline
\end{tabular}




\begin{tabular}{l|c|c|c|c} 
Continuą̧ão \\
\hline Higiene Pessoal & 16,7 & 83,3 & 0 & 100 \\
\hline Transferência & 33,3 & 66,7 & 40 & 60 \\
\hline Continência & 33,3 & 66,7 & 50 & 50 \\
\hline Alimentação & 33,3 & 66,7 & 100 & 0 \\
\hline
\end{tabular}

Ind.: Independentes. Dep.: Dependentes.

Observando os resultados da tabela acima nota-se que nas ABVD, transferência, continência e alimentação ocorreram melhoras, ou seja, na capacidade de transferir-se de um local para outro houve melhora de 6,7\%, na continência observou-se uma melhora de $16,7 \%$ e na capacidade para alimentar-se de $66,7 \%$, sendo esta última estatisticamente significativa ( $p$ $=0,025)$. Nas demais variáveis, banho, vestuário e higiene pessoal, não houve nenhuma mudança significativa, tendo uma tendência a aumentar o grau de dependência dos mesmos.

Com relação à aptidão física, esta foi avaliada considerando as seguintes variáveis: força de membros superiores (FMS) e de força de membros inferiores (FMI), flexibilidade de membros superiores (Flex MS) e inferiores (Flex MI), resistência aeróbica (RA) e agilidade e equilíbrio dinâmico (AED). Os resultados foram classificados em três categorias, abaixo do normal, normal e acima do normal, conforme classificação proposta por Rikli e Jones (2008) e estão apresentados na Tabela 2.

Tabela 2 - Aptidão física de idosos.

\begin{tabular}{|c|c|c|c|c|}
\hline \multirow{3}{*}{ Variáveis } & \multicolumn{4}{|c|}{ Classificação } \\
\hline & \multicolumn{2}{|c|}{ Pré-teste } & \multicolumn{2}{|c|}{ Pós-teste } \\
\hline & $\begin{array}{l}\text { Ab. do normal } \\
(\%)\end{array}$ & $\begin{array}{c}\text { Normal } \\
(\%)\end{array}$ & $\begin{array}{l}\text { Ab. do normal } \\
(\%)\end{array}$ & $\begin{array}{c}\text { Normal } \\
(\%)\end{array}$ \\
\hline FMS & 80 & 20 & 100 & 0 \\
\hline $\mathrm{FMl}$ & 80 & 20 & 100 & 0 \\
\hline Flex MS & 75 & 25 & 75 & 25 \\
\hline Flex Ml & 100 & 0 & 100 & 0 \\
\hline RA & 100 & 0 & 100 & 0 \\
\hline EAD & 100 & 0 & 100 & 0 \\
\hline
\end{tabular}

Ab. do normal: Abaixo do normal.

Após a análise de dados, constatou-se que nenhum dos participantes do estudo obteve classificação acima do normal, tanto no pré como no pós-teste. Ao analisar os valores da aptidão física em termos de média, foi possível observar 
melhoras na resistência aeróbica, na flexibilidade de membros inferiores e na flexibilidade de membros superiores.Entretanto, somente foi significativa, estatisticamente, esta melhora na variável flexibilidade de membros superiores $(\mathrm{p}=0,012)($ Tabela 3$)$.

Tabela 3 - Valores médios da aptidão física de idosos. Cruz Alta,RS, 2010.

\begin{tabular}{l|c|c|c}
\hline \multirow{2}{*}{ Variáveis } & Pré-teste & Pós-teste & \multirow{2}{*}{$\mathbf{P}$} \\
\cline { 2 - 3 } & Média \pm Desvio padrão & Média \pm Desvio padrão & \\
\hline FMl & $4,75 \pm 2,21$ & $4,83 \pm 2,48$ & 0,445 \\
\hline FMS & $8,75 \pm 6,23$ & $7,50 \pm 2,38$ & 0,119 \\
\hline RA & $197,00 \pm 102,56$ & $216,80 \pm 119,25$ & 0,376 \\
\hline Flex Ml & $-21,25 \pm 15,39$ & $-14,50 \pm 15,26$ & 0,867 \\
\hline Flex MS & $-28,00 \pm 33,04$ & $-2,25 \pm 4,78$ & 0,012 \\
\hline AED & $18,20 \pm 7,12$ & $21,50 \pm 13,10$ & 0,118 \\
\hline
\end{tabular}

Ab. do normal: Abaixo do normal.

A melhora da Flex MS pode estar relacionada às atividades que eram oferecidas aos idosos, considerando que até mesmo nas atividades menos ativas como as cognitivas (memória, pintura, jogos didáticos) os membros superiores estavam em movimento, pois se desenvolveram atividades de coordenação motora fina. Essa melhora observada na flexibilidade pode, também, estar relacionada com o encorajamento que os cuidadores passavam para eles, pois o grupo interdisciplinar que trabalhava com os cuidadores reforçava sempre a importância de tentar manter o máximo de tempo possível à independência dos idosos. A autonomia dos idosos foi correlacionada (Pearson) com as variáveis da aptidão física (Tabela 4).

Tabela 4 - Correlação lienar de Pearson (r) e Significância (p). Cruz Alta, RS, 2010.

\begin{tabular}{c|c|c|c|c|c|c|c}
\hline \multicolumn{2}{c|}{} & Banho & Vestuário & Higiene & $\begin{array}{c}\text { Transfe- } \\
\text { rência }\end{array}$ & $\begin{array}{c}\text { Conti- } \\
\text { nência }\end{array}$ & Ali. \\
\hline \multirow{2}{*}{$F M l$} & $r$ &, 437 &, 187 &,- 171 &, 465 &, $624^{*}$ &, 268 \\
\cline { 2 - 8 } & $P$ &, 207 &, 604 &, 661 &, 207 &, $050^{*}$ &, 454 \\
\hline \multirow{2}{*}{ FMS } & $r$ &, 468 &, 576 &, 476 &, 261 &, 057 &, 351 \\
\cline { 2 - 8 } & $P$ &, 242 &, 135 &, 280 &, 571 &, 893 &, 394 \\
\hline
\end{tabular}




\begin{tabular}{|c|c|c|c|c|c|c|c|}
\hline \multicolumn{8}{|c|}{ Continuaçāo } \\
\hline \multirow{2}{*}{ RA } & $r$ &, 412 & 317 & ,004 &, 589 & 893 & ,205 \\
\hline & $P$ &, 271 & ,407 & ,992 &, 125 & ,040 & ,596 \\
\hline \multirow{2}{*}{$\begin{array}{l}\text { Flex } \\
\text { Ml }\end{array}$} & $r$ & ,611 & 174 &,- 123 &,- 134 &, 224 &,- 516 \\
\hline & $P$ &, 108 & 680 &, 792 &, 774 &, 593 & 190 \\
\hline \multirow{2}{*}{$\begin{array}{l}\text { Flex } \\
\text { MS }\end{array}$} & $r$ &, $978^{*}$ & 653 & ,292 &,- 018 &,- 256 &,$- 739^{*}$ \\
\hline & $P$ &, 000 & ,112 &, 574 & ,973 &, 580 & ,048 \\
\hline \multirow{2}{*}{ Agil } & $r$ &,- 286 & 010 & , 144 &,- 513 &,- 569 &,- 104 \\
\hline & $P$ &, 456 & 980 & .733 & , 193 &, 110 & ,790 \\
\hline
\end{tabular}

Ali.: Alimentação. ** $p>0,01 e^{*} p>0,05$

Observando os dados da Tabela 4, notou-se associação significativa positiva moderada entre a força de membros inferiores e a resistência aeróbica com a continência. Também, observou-se uma forte correlação entre a flexibilidade de membros superiores com o banho e a alimentação.

Como se utilizam basicamente os membros superiores para alimentar-se e banhar-se, considerando que ambas as atividades podem ser realizadas sentadas, era de se esperar tal relação. As valências físicas força de membros inferiores e a resistência aeróbica contribuem para deambular e, diante disso, essas valências estão interligadas com a continência, pois no momento em que o idoso sente necessidade fisiológica, este tem condição de se encaminhar ao banheiro. Infelizmente, não foram encontrados estudos na literatura que relacionassem essas variáveis.

\section{Discussão}

Em banho, vestuário e higiene pessoal, todos os participantes tornaram-se totalmente dependentes de seus cuidadores, ou seja, as atividades propostas não repercutiram de forma favorável nem para a manutenção da independência dos idosos que a apresentavam antes de ingressar no projeto. Cabe salientar aqui que sete meses de evolução da doença são suficientes para os idosos apresentarem uma grande evolução do quadro da demência. Embora a DA apresente prognóstico de evolução variando entre três a dez anos, é importante ressaltar que a progressão dela depende fundamentalmente de cada indivíduo, pois o estilo de vida de cada um repercute diretamente na sua evolução (ARAÚJO; NICOLI, 2010).

Os dados do presente estudo são semelhantes aos obtidos por Oliveira, Goretti e Pereira (2006), que também utilizaram Katz na sua pesquisa. Esses autores mostraram que os idosos com Alzheimer têm dificuldades com relação 
a banho, vestuário e transferência. Isso se deve à dificuldade de mobilidade e que as alterações cognitivas não influenciaram tanto nestas atividades.

Um estudo realizado no Centro de Referência em DA do Município de Curitiba, Paraná, por Lenardt et al. (2011), cujo objetivo foi avaliar o desempenho das atividades básicas de idosos com Alzheimer, evidenciou que 56,4\% eram semidependentes por terem uma das atividades diárias comprometidas. Entretanto, com relação à transferência e à continência, dos 55 participantes, 43 foram classificados como independentes, totalizando assim 78\%. Esses dados discordam do presente estudo, pois segundo Lenardt et al. (2011), 78\% dos participantes eram independentes nas atividades básicas diárias de transferência e continência, diferente deste estudo, em que os números permearam entre $40 \%$ na independência de transferência e 50\% de independência na continência.

Em outro estudo realizado por Hassen et al. (2006), que teve um total de 11 participantes, devidamente diagnosticados com a doença de Alzheimer, somente três pacientes eram independentes para alimentação, dois para transferência e uso do banheiro e um para continência. Todos eram dependentes para banho e vestuário, tendo assim, como resultado, um elevado grau de dependência, indo ao encontro dos resultados deste estudo.

Com relação à alimentação, é importante ressaltar que inicialmente o percentual de dependentes parciais e total era elevado (66,7\%) e, após as intervenções, todos se tornaram independentes. Comparando com os resultados do estudo de Hassen et al. (2006), nota-se que não houve semelhanças, pois no referido estudo de 11 idosos, somente 3 eram independentes.

Cabe salientar que a melhora significativa com relação à alimentação pode ter sido fruto das conversas de conscientização com os cuidadores, realizadas durante os encontros, sempre que se observava que os mesmos auxiliavam os idosos, mesmo alguns tendo condições para realizar essa função. A conscientização deu-se no sentido de deixar que os idosos realizassem essa função mesmo demorando mais, pois assim estaria contribuindo para minimizar a dependência do idoso. A maioria dos cuidadores relatou que faziam isso devido ao longo tempo e à sujeira que os idosos faziam durante as refeições.

Segundo Talmelli (2009), é importante diagnosticar o nível de dependência dos idosos com Alzheimer para que assim seja possível traçar um plano de cuidados juntamente com a família e/ou cuidadores para que estes estejam preparados para melhor enfrentar a progressão da doença. É relevante, também, conscientizar os cuidadores para, sempre que possível, estimular a independência dos idosos, pois assim estes terão uma melhor qualidade de vida.

Como no final dos encontros, havia o momento do lanche, e era visível a ajuda que os cuidadores davam a eles. Assim, toda vez que os cuidadores 
os ajudavam, era salientada a importância de deixar os idosos realizarem a atividade sozinhos sempre que possível. Como afirma Borges (2011), toda atividade realizada pelo idoso com a doença de Alzheimer deve ser sempre orientada e observada pelo seu cuidador, mas este deve sempre propiciar a maior autonomia possível ao idoso, visando assim gerar maior tempo de independência do idoso com relação ao seu cuidador.

Para Benedetti et al. (2008), quanto maior o tempo de atividade física que um idoso realiza, melhor será seu desempenho nas atividades básicas diárias, pois a relação que se estabelece entre a atividade física e a demência é de que quanto mais se pratica, mais se consegue reduzir e/ou atrasar os riscos da demência. Entretanto, a prática da atividade física não assegura que a demência não surja. Embora a melhora na resistência aeróbica não tenha sido significativa estatisticamente, ela aumentou em $25 \%$ do pré para o pós-teste, ou seja, a média geral do grupo aumentou de 197 metros no pré-teste para 216,80 metros no pós-teste.

Cabe salientar aqui que os encontros do projeto aconteceram quinzenalmente e que todos os idosos do projeto somente faziam atividades físicas nos referidos encontros, mesmo os cuidadores tendo recebido orientações e estímulo para realizarem caminhadas e alongamentos com os idosos em casa. Como se sabe, a prática de exercícios físicos esporadicamente não contribui eficazmente para a aptidão física e mesmo assim observou-se melhoras em algumas das variáveis estudadas. Outro fator que deve ser observado é que alguns idosos não realizaram ou interromperam os testes antes de término devido aos problemas próprios da doença e não necessariamente por diminuição da aptidão física.

Entretanto, é importante salientar que, com a progressão da doença, há uma diminuição da aptidão física e com isso o idoso se torna mais dependente de seu cuidador. Segundo Marra et al. (2007), à medida que aumenta a gravidade da demência mais comprometida fica à execução das atividades básicas do cotidiano. Primeiramente, perde-se a capacidade de realizar atividades mais complexas, como cuidar das finanças, fazer compras, preparar as refeições, lembrar-se do horário e do modo de usar os medicamentos e cuidar da casa. Posteriormente, apresentam dificuldades para banhar-se, vestir-se, usar o vaso sanitário e, ao final, alimentar-se, ficando totalmente dependente de seu cuidador.

Marra et al. (2007) salientam que as alterações no comportamento, como irritabilidade, ansiedade, apatia e depressão, acompanham a evolução da demência e, lenta e gradualmente, ocorrem mudanças no comportamento habitual (personalidade), perdendo-se o senso crítico. Essas alterações de 
comportamento e conduta podem estar ligadas ao ambiente, a mudanças bruscas de costumes e a situações estressantes. Salientam ainda os mesmos autores que as reações são diversas, desde inquietação e xingamentos até agressão física.

No momento da aplicação dos testes, alguns deles apresentaram o comportamento citado acima, pois o local de aplicação dos mesmos era um ambiente diferente do qual estavam habituados, saindo assim da sua rotina. Alguns tiveram de realizar os testes com o cuidador ao lado para que assim fosse possível concluí-los, enquanto outros idosos nem mesmo com a presença do cuidador quiseram voltar a realizá-los.

Segundo Neves (2009), a força muscular possibilita a execução de várias atividades no cotidiano. Além disso, uma característica de sua perda está mais relacionada com a diminuição de atividade física do que com a idade. Santarém (2000) salienta que o treino da força muscular é importante para o idoso manter a capacidade de realizar as tarefas cotidianas, que normalmente exigem muito mais de força muscular, resistência muscular e flexibilidade do que de capacidade aeróbia.

É importante salientar que a proposta do projeto que visa à autonomia e à reabilitação do idoso vem ao encontro das políticas de saúde propostas pelo Sistema Único de Saúde (SUS) e pela Política Nacional do Idoso (PNI), que preveem que o atendimento aos idosos deve ser prioridade da família em detrimento do atendimento asilar, prevendo também a capacitação e reciclagem dos recursos humanos nas áreas de geriatria e gerontologia e na prestação de serviços. Ainda, o artigo $1^{\circ}$ prevê que a PNI deve assegurar os direitos sociais do idoso, criando condições para promover a sua autonomia, integração e participação efetiva na sociedade. Além disso, o artigo $3^{\circ}$ enfoca ainda que a família, a sociedade e o Estado têm o dever de assegurar ao idoso todos os direitos de cidadania, garantindo sua participação na comunidade, defendendo sua dignidade, bem-estar e o direito à vida (BRASIL, 1994).

Entende-se que o projeto desenvolveu ações voltadas para o atendimento das necessidades básicas do idoso, mediante a participação das famílias e da sociedade, através da Universidade e de entidade governamental como a Prefeitura Municipal de Cruz Alta. No entanto, para que os resultados sejam mais efetivos, sugere-se realizar encontros semanais ou de duas vezes por semana.

Após análise dos dados, foi possível concluir que tomar banho, fazer sua higiene pessoal e vestir-se são as atividades básicas da vida diária que os idosos apresentaram maior dificuldade de realização com a progressão da 
doença. O programa de atividades propostas aos idosos proporcionou uma melhora na autonomia em relação à continência, à transferência e à melhora estatisticamente significativa na autonomia para alimentar-se.

Com relação à aptidão física, percebeu-se que nem no pré e nem no pós-teste os idosos conseguiram atingir a classificação acima do normal. Apesar de os idosos se manterem abaixo do normal nos testes, a participação nas atividades propostas pelo projeto influiu de forma significativa na variável flexibilidade de membros superiores $(\mathrm{p}=0,012)$.

Sendo assim, se levarmos em conta que $100 \%$ dos idosos tornaram-se independentes para sua alimentação e que a flexibilidade de membros superiores teve melhora estatisticamente significativa, fica evidente que outras variáveis poderão ser melhoradas futuramente, aumentando o número de encontros semanais para a prática de exercícios físicos. Em paralelo, é recomendado orientar os cuidadores para que permitam que os idosos realizem suas atividades com o menor auxílio possível, incentivando sua autonomia e independência.

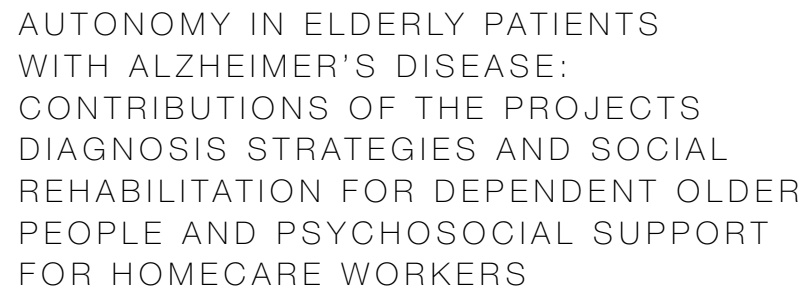

\section{abstract}

This study analyzed the contributions of conducting therapeutic activities in psychosocial and functional autonomy and fitness of six elderly with Alzheimer's disease. To evaluate the independence used the Scale ADL-Katz. The physical fitness through the battery of tests Rikli and Jones. The meetings with the group of seniors and caregivers occurred biweekly for a total of 12 sessions. Psychosocial and functional therapeutic activities consisted of stretching, walking and dancing. This session lasted approximately 40 minutes after the elderly held diversified activities through Memorization educational games, puzzles games, fine motor skills and manual dexterity, and sensory activities involving taste, touch and smell. The data were interpreted and described by descriptive statistical inference, and percentage. After analyzing the data, we observed improvements, pre to post-test, the following variables: ability to transfer from one location to another, at $6.7 \%$, continence, $16.7 \%$, and the ability to feed, $66.7 \%$, 

the classification for seniors. Participation in the activities proposed by the project influenced significantly ( $p=0.012)$ only in the flexibility of the upper limbs. Thus, it was concluded that a physical activity program for seniors with Alzheimer's, is very important, however there should be more regular sessions for the results to be more relevant.

\section{keywords}

Alzheimer's Disease. Motor Activity. Physical Fitness.

\section{referências}

ABREU, Izabella Dutra; FORLENZA, Orestes Vicente; BARROS, Hélio Lauar. Demência de Alzheimer: correlação entre memória e autonomia. Revista de Psiquiatria Clínica, São Paulo, v. 32, n. 3, p. 131-136, maio/jun. 2005.

ALVES, José Eustáquio Diniz. Transição demográfica, transição da estrutura etária e envelhecimento. Revista Portal de Divulgação, São Paulo, n. 40, ano IV. p. 8-15, mar./ abr./maio 2014.

ALZHEIMER'S DISEASE INTERNATIONAL. Relatório sobre a Doença de Alzheimer no mundo de 2009. Resumo Executivo. Disponivel em: <http://www.alz.co.uk/research/ files/WorldAlzheimerReport-Portuguese.pdf.>. Acesso em: 23 jul. 2011.

ARAÚJO, Claudia Lysia de O.; NICOLI, Juliana Silva. Uma revisão bibliográfica das principais demências que acometem a população brasileira. Kairós, São Paulo, v. 13, n. 1, p. 231-244, jun. 2010.

ARRUDA, Michel Coral; ALVAREZ, Ângela Maria; GONÇALVES, Lucia Hasako Takase. O familiar cuidador de portador de doença de Alzheimer participante de um grupo de ajuda mútua. Ciência, Cuidado e Saúde, Maringá, v. 7, n. 3, p. 339-345, jul./set. 2008.

BANDEIRA, Denise Ruschel; GONÇALVES, Tonantzin Ribeiro; PAWLOWSKI, Josiane. Envelhecimento e dependência: impacto sobre familiares-cuidadores de portadores de síndrome demencial. In: PARENTE, Maria Alice de Mattos Pimenta (Org.). Cognição e Envelhecimento. Porto Alegre: Artmed, 2007. p. 275-284.

BORGES, Márcio Fernando. Convivendo com Alzheimer: Manual do Cuidador de Alzheimer. Minas Gerais: ABRAZ, 2011. Disponível em: <http://www.cuidardeidosos. com.br/wp-content/uploads/2008/04/manual-do-cuidador-alzheimer.pdf>. Acesso em: 23 jul. 2011.

BRASIL. Lei 8.842, de 4 de janeiro de 1994. Dispõe sobre a Política Nacional do Idoso, cria o Conselho Nacional do Idoso e dá outras Providências. Diário Oficial [da] República Federativa do Brasil. Brasília, DF, 5 jan. 1994.

CINTRA, Marco Túlio Gualberto et al. Avaliação do programa público brasileiro de tratamento da doença de alzheimer, no ano de 2008. Governo do Estado de São Paulo. Disponível em: <http://www.saude.sp.gov.br/resources/ses/perfil/profissional-da-saude/ grupo-tecnico-de-acoes-estrategicas-gtae/saude-da-pessoa-idosa/encontro-nacional-de-coordenadores-de-saude-da-pessoa-idosa/avaliacao_do_programa_publico_brasileiro_de_tratamento_da_doenca_de_alzheimer_no_ano_de_2008.pdf $>$. Acesso em: 10 maio 2012. 
COUTINHO FILHO, Roque Crispim. As influências da prática de atividade física nas funções cognitivas em idosos. Lecturas: Educación Física y Deportes, Buenos Aires, año 12, n. 118, p. 1-1, marzo 2008

FARINATTI, Paulo de Tarso Veras. Envelhecimento: promoção da saúde e exercícios. São Paulo: Manole, 2008. 499 p.

GARCES, Solange Beatriz Billig et al. Estratégias de diagnóstico, reabilitação social de idosos com Alzheimer e apoio psicossocial ao cuidador: uma experiência na Unicruz. Cataventos, Cruz Alta, n. 2, p. 1-18, 2010.

HASSEN, Vivian Garcia et al. Perfil funcional de portadores da doença de Alzheimer na enfermaria de geriatria do Hospital do Servidor Público Estadual "Francisco Morato de Oliveira". Revista Médica do IAMSPE, São Paulo, v. 31, n. 4, p. 189-193, 2006.

INSTITUTO BRASILEIRO DE GEOGRAFIA E ESTATÍSTICA. Censo Demográfico: resultados preliminares - 2010. Disponivel em: <<http://www.ibge.gov.br/home/estatistica/ populacao/censo2010/resultados_preliminares_amostra/notas_resultados_preliminares_amostra.pdf>. Acesso em: 15 maio 2012.

LENARDT, Maria Helena et al. Desempenho das atividades de vida diária em idosos com Alzheimer. Cogitare Enfermagem, Curitiba, v. 16, n. 1, p. 13-21, jan/mar. 2011.

MARRA, Taís de Almeida et al. Avaliação de atividades de vida diária de idosos com diferentes níveis de demência. Revista Brasileira de Fisioterapia, São Carlos, v. 11, n. 4, p. 267-273, jul/ago. 2007.

MATSUDO, Sandra Marcela Mahecha. Envelhecimento, atividade física e saúde. Boletim do Instituto de Saúde, São Paulo, n. 47, p. 75-78, abr. 2009.

NEVES, Luís Pedro Gomes. Caracterização dos parâmetros morfológicos e da aptidão física em idosos do Concelho de Coimbra: sua relação com a qualidade de vida. 2009. 89 f. Dissertação (Licenciatura em Educação Física) - Faculdade de Ciências do Desporto e Educação Física, Universidade de Coimba, Coimbra, 2009. Disponíve em: <http://hdl.handle.net/10316/11998>. Acesso em: 27 jun. 2012.

OLIVEIRA, Debora Lopes Costa; GORETTI, Luciane Correa; PEREIRA, Leani Souza Máximo. O desempenho de idosos institucionalizados com alterações cognitivas em atividades de vida diária e mobilidade: estudo piloto. Revista Brasileira de Fisioterapia São Carlos, v. 10, n. 1, p. 91-96, jan/mar. 2006.

OLIVEIRA, Maria de Fátima et al. Doença de Alzheimer: perfil neuropsicológico e tratamento. 2005. 21 f. Trabalho de Conclusão de Curso (Licenciatura em Psicologia em Saúde) - Departamento de Psicologia, Universidade Lusíada do Porto, Porto, 2005

ORGANIZAÇÃO DAS NAÇÕES UNIDAS. A ONU e as pessoas idosas. Relatório da Organização das Nações Unidas, 2011. Disponível em: <http://nacoesunidas.org/acao/ pessoas-idosas>. Acesso em: 7 maio 2014.

PEREIRA, Márcio de Moura; SAFONS, Marisete Peralta. Metodologia da Dança de Salão para Idosos. In: SAFONS, Marisete Peralta; PEREIRA, Márcio de Moura (Org.). Educação Física para Idosos: por uma prática fundamentada. 2. ed. Brasília: CREF/ DF- FEF/UnB/GEPAFI, 2007. p. 165-167.

RIKLI, Roberta; JONES, Jessie. Teste de Aptidões Físicas para Idosos. Barueri: Manole 2008. p. 183

SANTARÉM, José Maria. Qualidade dos exercícios resistidos. Saúde Total, 19 maio 1999. Disponivel em: <http://www.saudetotal.com.br/artigos/atividadefisica/qualidade. asp>. Acesso em: 14 abr. 2011

SANTOS, Ariene Angeline dos; PAVARINI, Sofia Cristina lost. Perfil dos cuidadores de idosos com alterações cognitivas em diferentes contextos de vulnerabilidade social. Revista Gaúcha de Enfermagem, Porto Alegre, v. 31, n. 1, p. 115-122, mar. 2010. 
Recebido: 03/06/2013

Aceite Final: 16/11/2015
SCHLINDWEIN-ZANINI, Rachel. Demência do idoso: aspectos neuropsicológicos. Revista Neurociências, São Paulo, v. 18, n. 2, p. 220-226, abr./maio 2010.

TALMELLI, Luana Flávia da Silva. Nivel de independência funcional de idosos com Doença de Alzheimer. 2009. 113 f. Dissertação (Mestrado em Enfermagem) - Escola de Enfermagem de Ribeirão Preto, Universidade de São Paulo, Ribeirão Preto, 2009.

VIEIRA, Renata Teles; CAIXETA, Leonardo. Epidemiologia da Doença de Alzheimer. In: CAIXETA, Leonardo et al. Doença de Alzheimer. Porto Alegre: Grupo A, 2012.

WORLD HEALTHORGANIZATION. Dementia: a public health priority. Geneva: WHO, 2012. Disponivel em: <http://apps.who.int/iris/bitstream/10665/75263/1/9789241564458_eng. pdf?ua=1> Acesso em: 22 set. 2014.

ZIDAN, Melissa et al. Alterações motoras e funcionais em diferentes estágios da doença de Alzheimer. Revista de Psiquiatria Clínica, São Paulo, v. 39, n. 5, p. 161-165, 2012. 